\title{
Nacidos vivos con bajo peso al nacer en Bogotá 2014-2016.
}

\author{
Alejandro Avelino B ${ }^{1}$, Yeraldim A. Coronado $\mathrm{G}^{1}$, Felipe E. Hernández R ${ }^{1 *}$. \\ ${ }^{1}$ Especialistas en Epidemiologia, Médicos Generales, Fundación Universitaria Juan N. Corpas. Bogotá Colombia.
}

\begin{abstract}
RESUMEN
Introducción. La Organización Mundial de la Salud OMS considera el bajo peso al nacer como un problema de salud pública, dado que esta entidad aumenta el riesgo de padecer enfermedades no transmisibles en etapas posteriores de la vida y es la causa de morbilidad y mortalidad neonatal más frecuente. El bajo peso al nacer trae consigo deficiencia en el desarrollo físico y neurológico del neonatal, tanto a largo como a corto plazo, sea por patologías congénitas, genéticas o por eventos secundarios a la internación en unidades de cuidados intensivos neonatales, tales como infecciones asociada al cuidado de la salud, uso de ventilación asistida, consumo de determinados fármacos $\mathrm{u}$ otras condiciones que van sujetas a hospitalizaciones prolongadas al recién nacido.

Objetivos. Medir la frecuencia de bajo peso al nacer de gestaciones a término y pretérmino e identificar los posibles factores de riesgo relacionados.

Metodología. Se realizó un estudio descriptivo retrospectivo en nacimientos ocurridos en la ciudad de Bogotá entre los años 2014 a 2016, basados en datos recolectados por el Departamento Administrativo Nacional de Estadística DANE, utilizando medidas de tendencia central para evaluar la frecuencia de peso al nacer bajo.

Resultados. El total de nacidos vivos en el periodo estudiado, según las estadísticas vitales del Departamento Administrativo Nacional de Estadística fue de 349.967 , de los cuales 44.817 fueron nacidos vivos con bajo peso, lo que equivale al $12,80 \%$ del total de la población. El mayor registro se documentó en el año 2014 con 14.973 casos; las gestantes en las edades extremas, menores de 15 años y mayores de 44 años tienen las tasas más elevadas de bajo peso al nacer 0,18 y 0,25 respectivamente.

Conclusiones. Las edades maternas extremas, resulta ser un factor riesgo para desarrollar bajo peso al nacer el intervalo de bajo peso al nacer más frecuente durante el periodo estudiado fue entre los $2.000 \mathrm{y}$ 2.499 gr. El único año en el que se identificó diferencia estadística fue 2016, las edades maternas extremas presentaron las tasas más altas de nacidos vivos con bajo peso fueron en las localidades de Suba y Kennedy.
\end{abstract}

Palabras clave: Nacido vivo, peso al nacer, natalidad, parto, orden de nacimiento.

\section{ABSTRACT}

\section{Born alive with low weight at birth in Bogotá 2014-2016.}

Introduction. The World Health Organization (WHO) considers low birth weight a public health problem, since it increases the risk of noncommunicable diseases in later life and is the most frequent cause
\end{abstract}

of neonatal morbidity and mortality. Low birth weight brings with it a deficiency in the physical and neurological development of the neonatal, both in the long and short term, either due to congenital or genetic pathologies or due to events secondary to hospitalization in neonatal intensive care units, such as infections associated with health care, use of assisted ventilation, use of certain drugs or other conditions that are subject to prolonged hospitalization of the newborn.

Objective. To measure the frequency of low birth weight in term and preterm pregnancies and to identify possible related risk factors.

Methodology. A retrospective descriptive study was carried out on births that occurred in the city of Bogotá between the years 2014 and 2016, based on data collected by the National Administrative Department of Statistics (DANE), using central tendency measures to evaluate the frequency of low birth weight.

Results. The total number of live births in the period studied, according to the vital statistics of the National Administrative Department of Statistics was 349.967, of which 44.817 were live births with low weight, equivalent to $12,80 \%$ of the total population. The largest record was documented in 2014 with 14.973 cases; pregnant women in the extreme ages, under 15 and over 44, have the highest rates of low birth weight 0,18 and 0,25 respectively.

Conclusions. Extreme maternal ages are a risk factor for developing low birth weight. The most frequent low birth weight interval during the period studied was between 2,000 and 2,499 grams. The only year in which a statistical difference was identified was 2016, the extreme maternal ages presented the highest rates of live births with low weight were in the towns of Suba and Kennedy.

Keywords: Live birth, birth weight, natality, childbirth, birth order. Felipe E. Hernández Romero felipe-hernandez@juanncorpas.edu.co

Como citar: Avelino Bonilla A, Coronado Gutiérrez YA, Hernández Romero FE. Nacidos vivos con bajo peso al nacer en Bogotá 2014-2016. Revista Cuarzo 2020;26(2):7-10.

Recibido: 12 de noviembre de 2020

Aceptado: 2 de diciembre de 2020

Publicado: 30 de diciembre de 2020

DOI: https://doi.org/10.26752/cuarzo.v26.n2.512 


\section{INTRODUCCIÓN}

T a organización Mundial de la salud (OMS) define el bajo

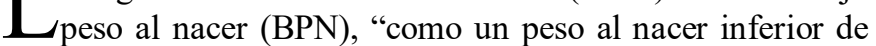
2500 gr" (1), se estima que un porcentaje menor de los niños nacidos en todo el mundo son susceptibles de presentar bajo peso al nacer. El valor de llevar a cabo un abordaje investigativo es la importancia mundial de reducir el número de neonatos con bajo peso al nacer, ya que es la causa de morbilidad y mortalidad neonatal más frecuente (2).

Los porcentajes regionales en la distribución mundial según estimaciones de la OMS son del $28 \%$ en Asia meridional, el $13 \%$ en el África subsahariana y el 9\% en Latinoamérica para el 2013 (1), la proporción de niños con bajo peso al nacer oscila entre 5,20\% en Cuba y 23\% en países como Haití (3).

En Colombia, el Departamento Administrativo Nacional de Estadística (DANE) tiene a cargo el sistema de estadísticas vitales (nacimientos y defunciones) (4), se reconoce que para el 2014 el bajo peso al nacer ha presentado un descenso con relación a años anteriores, cifra que a pesar de ser menor frente a los cálculos realizados en el informe de Fondo de las Naciones Unidas para la Infancia (UNICEF) en 2016, es considerada alta si se compara con otros países de la región (5).

El bajo peso al nacer trae consigo deficiencia en el desarrollo físico y neurológico del neonato, tanto a largo como a corto plazo, sea por patologías congénitas, genéticas o por eventos secundarios a la internación en unidades de cuidados intensivos neonatales, tales como infecciones asociadas al cuidado de la salud, uso de ventilación asistida, consumo de determinados fármacos u otras condiciones que van sujetas a hospitalizaciones prolongadas (6).

La realización de esta investigación tiene como objetivos identificar las características demográficas y sociales de la población en estudio, medir la frecuencia de bajo peso al nacer tanto en recién nacidos productos de gestaciones a término como pretérmino en la ciudad de Bogotá, identificar los posibles factores de riesgo relacionados con el bajo peso al nacer, establecer las principales características de las gestantes que pueden desencadenar nacimientos con bajo peso e identificar la diferencia estadística entre presencia del bajo peso al nacer de productos de una gestación a término y pretérmino.

\section{MÉTODOS}

Estudio descriptivo retrospectivo, en todo nacido vivo con bajo peso, producto de la gestación de madres que registraron el nacimiento en la ciudad de Bogotá, durante los años 2014 a 2016, ya sean nacidos vivos de gestaciones antes del término como a término.

Las variables cualitativas nominales son: el sexo del nacido vivo, estado civil, tipo de afiliación, sitio de nacimiento sea en centros de salud o fuera de ellos, tipo de documento de identidad, el parto fue atendido por, pertenencia a etnia, último año de estudio de la madre y del padre, área de residencia, régimen de seguridad social, tipo de nacimiento sea vaginal o cesárea, multiplicidad del embarazo.

Las variables cuantitativas de razón son: edad materna, número de niños con bajo peso al nacer pre término y a término por mes, edad gestacional, número de controles prenatales, número de embarazos de la madre. peso del nacido vivo, talla del nacido vivo.

Los datos se consolidaron en un documento de Excel, obtenido de las fuentes estadísticas nacionales provistas públicamente por el Departamento Administrativo Nacional de Estadística (DANE), registrados en la página web en la sección de estadísticas vitales, de la que se seleccionaron los registros almacenados durante los años 2014 al 2016. Estos datos son el resultado del diligenciamiento de los certificados de nacido vivo, los cuales son almacenados en la página en mención.

A los datos obtenidos se les calcularon distribuciones de frecuencias, que se presentaron en tablas y gráficos, medidas de tendencia central tales como la media, moda, mediana; a las variables numéricas se les calcularon las medidas de dispersión. Los resultados principales de Bajo peso al nacer fueron presentados en proporciones e incidencias durante los años que fueron estudiados.

Se realizó una investigación sin riesgo, según la resolución 8430 de 1993 del Ministerio de Educación, dado que la información fue obtenida de bases de datos sin ningún tipo de intervención con la población estudiada. Se garantiza confidencialidad, así mismo la información fue utilizada únicamente con fines informativos y los datos no fueron utilizados antes de que el proyecto hubiera estado aprobado.

\section{RESULTADOS}

Durante los tres años que fueron estudiados, el bajo peso al nacer solo tuvo diferencia estadística en el año 2016, tomando como referente el año 2015. Dentro de las estadísticas vitales del DANE en los años de 2014 a 2016 fue de 349.967, de los cuales 44.817 fueron nacidos vivos con bajo peso, lo que equivale al $12,80 \%$ del total de la población registrada. El mayor registro se documentó en el año 2014 con 14.973 y el menor en el año 2016 con 14.917 nacidos vivos con bajo peso.

El American College of Obstetricians and Gynaecologists (ACOG) propone una nueva clasificación del embarazo a término, en la cual los embarazos entre 37 semanas 0/7 días y 38 semanas 6/7 días se denominan embarazos a término temprano; los que tienen entre 39 semanas 0/7 días y 40 semanas 6/7 días son embarazos a término; los de 41 semanas 0/7 días y 41 semanas 6/7 días, término tardío, y los de 42 semanas 0 días y más, postérmino (7) véase en la Tabla 1 la aproximación de los intervalos de gestación respectivos.

Para el 2014 se comprobó que de 1000 nacidos vivos 0,25 tienen bajo peso al nacer en gestantes entre los 45 y 49 años, 
tasas similares se obtuvieron en los años siguientes en el mismo grupo de edad; seguida de una tasa de 0,18 por cada 1000 nacidos vivos con bajo peso al nacer en gestantes entre los $10 \mathrm{y}$ 14 años en el año 2014, así como tasas similares en los años siguientes.

Tabla 1: Incidencia del tiempo de gestación (semanas- ACOG) en nacidos vivos con bajo peso en Bogotá, 2014-2016.

\begin{tabular}{rllllll}
\hline \multicolumn{1}{c}{$\begin{array}{c}\text { Semanas de } \\
\text { gestación }\end{array}$} & \multicolumn{2}{c}{$\mathbf{2 0 1 4}$} & \multicolumn{2}{c}{$\mathbf{2 0 1 5}$} & \multicolumn{2}{c}{$\mathbf{2 0 1 6}$} \\
Caso & Tasa & Caso & Tasa & Caso & Tasa \\
\hline \hline 22 & 6 & 1,0 & 5 & 1,0 & 4 & 1,0 \\
$22-27$ & 552 & 0,97 & 505 & 0,96 & 543 & 0,99 \\
$28-37$ & 11.501 & 0,44 & 11.642 & 0,45 & 11.629 & 0,46 \\
$38-41$ & 2.903 & 0,03 & 2.766 & 0,03 & 2.734 & 0,03 \\
42 y más & 1 & 0,01 & 3 & 0,04 & 0 & 0 \\
Sin información & 10 & 0,32 & 6 & 0,22 & 7 & 0,21 \\
\hline \hline Total & $\mathbf{1 4 . 9 7 3}$ & $\mathbf{0 , 1 2}$ & $\mathbf{1 4 . 9 2 7}$ & $\mathbf{0 , 1 2}$ & $\mathbf{1 4 . 9 1 7}$ & $\mathbf{0 , 1 3}$ \\
\hline \hline Fuente: Departamento Administrativo Nacional de Estadísticas - Estadísticas vitales - nacimientos- \\
\multicolumn{7}{c}{ 2014-2016. }
\end{tabular}

Como dato importante encontrado en el análisis global de las cifras suministradas los altos números de nacimientos en el año 2014 fue en la Localidad de Kennedy con 15.024 en total con 1780 nacimientos catalogados con bajo peso al nacer, en segundo lugar, continua Suba con 14. 749 con 1.884 nacimientos con bajo peso al nacer, en contraste la menor localidad con nacimientos este año fue Sumapaz con 28 nacimientos de los cuales 6 fueron de bajo peso al nacer en Bogotá.

En el año 2015 se identificaron 11.642 nacimientos con bajo peso entre la semana 28 y 37 de gestación. Durante los años 2014 y 2015 en la ciudad de Bogotá de 1.000 nacidos vivos con bajo peso uno tiene menos de 22 semanas de gestación y para el año 2014 se determinó que 0,01 de cada 1.000 nacidos vivos con bajo peso tiene de 42 a más semanas de gestación.

El resultado de la tasa en el año 2014 se encontró como referente la localidad de Puente Aranda con 0.110 nacidos vivos con bajo peso al nacer por cada 1000 habitantes en la localidad a comparación del resto de territorio en Bogotá D.C. Para los años 2015-2016 la localidad de Santafé presentó la cifra como referente para analizar los datos de los años en mención. Se recuerda que el asocio entre variables estudiadas y hallazgos puede variar dado que el DANE solo toma el dato, sin poder evaluar impacto, se puede inferir en que la clasificación de gestación no se encontró una definición global para generar un concepto favorable para tener una precisión básica y con el universo total del mismo.

\section{DISCUSIÓN}

Desde el año 2014 al 2016 en la ciudad de Bogotá, según las estadísticas vitales del Departamento Administrativo Nacional de Estadísticas, del total de nacidos vivos el 12,80\%. Estos resultados van de la mano con lo documentado en las literaturas, donde se especifica que las gestaciones múltiples pueden desencadenar parto pretérmino y consecuentemente bajo peso al nacer, ya sea por insuficiencia placentaria o por el síndrome de transfusión feto fetal, situación que acrecienta la incidencia del evento al aumentar el número de fetos involucrados $(8,9)$.

Entre los factores psicosociales que se encuentra asociados a bajo peso al nacer y sus consecuentes complicaciones, son los bajos recursos económicos, las limitaciones para lograr acceder de forma rutinaria al control prenatal, la desinformación en cuanto a la consulta pre concepcional y de nutrición, puede estar relacionada con la no identificación temprana y oportuna de enfermedades maternas y fetales, tales como infecciones congénitas, anomalías congénitas, trastorno hipertensivos en la gestante, desnutrición o síndrome de mala absorción (8), defectos en la placenta, que pueden desencadenar restricción en el crecimiento intrauterino y el consecuente bajo peso al nacer (9), así como el hecho de no realizar modificaciones nutricionales ni aumentar la ingesta de micronutrientes, no realizar cambios en hábitos que no son saludables, tales como el consumo de sustancias psicoactivas o fármacos y tabaco (9).

En cuanto al comportamiento del evento por quinquenio de edad materna, las gestantes en las edades extremas, menores de 15 y mayores de 44 años tienen las tasas más elevadas $(0,18$ y 0,25 respectivamente) situación que es concordante con lo descrito en las literaturas (1).

La menor incidencia de bajo peso al nacer se presentó en el quinquenio entre los 25 y 29 años, con una tasa de 0,11 por 1.000 nacidos vivos, confirmando que estas edades son las más seguras para evitar la aparición de bajo peso al nacer (2).

Con lo anterior se confirma que las edades extremas de la vida, en este caso menores de 14 años y mayores de 44, resultan ser un factor de riesgo para desencadenar este evento (11), ya sea porque la gestante no ha completado su propio proceso fisiológico de desarrollo y madurez o lo está finalizando y el organismo no se encuentra preparado para una gestación (11). Las mujeres mayores de 44 años se encuentran iniciando la etapa conocida como menopausia y la carga hormonal, así como las características de sus órganos reproductores (10), han sufrido cambios y atrofias que no son compatibles con una gestación y en caso de desencadenarse una, puede el producto de la gestación, así como la mujer tener complicaciones entre las que se encuentra el bajo peso al nacer (10).

La localización según las bases en Bogotá D.C. siempre presenta alguna variación desde cifras hasta el reporte de lugares en localidades (11), donde se debe diferenciar grandes territorios en las mismas que combinan el escenario rural y el urbano, donde nunca pueden ser relacionados con zonas en la cuidad mayor pobladas, otro dato de importancia es que el gran número de caso siempre son encontrados en las zonas centrales de la cuidad o donde se encuentran grandes hospitales especializados en salud materno perinatal, el gran inconveniente es saber el origen de las maternas, donde no es el lugar de nacimiento en la institución hospitalaria, sino su entorno y origen demográfico(11); que debe ser de vital importancia; de ahí se parte inicialmente que el reconocimiento 
geográfico de una gestante de bajo peso siempre son de zonas donde las barreras de un adecuado control prenatal, el sub registro de muchas fichas de notificación de bajo peso al nacer puede ser otro componente importante ya que muchos de estos productos de la gestación se encuentran en los traslados de unidad de cuidado critico neonatal en donde muchas veces manejan según disponibilidad de las misma (11), y siempre que ingresa un nuevo neonato se reporta, algo aún más importante que no se tiene evidencia de los casos es el seguimiento, un gran capitulo que debe importar y realmente se tiene poca evidencia en números y la progresión de estos niños y su crecimiento además de su desarrollo como niños sanos $(1,2)$.

\section{CONCLUSIONES}

El año en el cual se registraron más casos de bajo peso al nacer durante el periodo estudiado fue en el 2016.

Las edades maternas extremas, es decir las menores de 19 años y las mayores de 35 años tienen más riesgo de que sus nacidos vivos tengan bajo peso.

El intervalo de bajo peso al nacer más frecuente durante el periodo estudiado fue entre los 2.000 y $2.499 \mathrm{gr}$.

Las localidades más afectas en estos casos durante los 3 años fueron: la localidad de Suba y Kennedy.

Los factores de riesgo descritos en la literatura se presentaron como factores de riesgo en el estudio.

Conflicto de Interés: Los investigadores declaran no tener ningún conflicto de interés en la realización del estudio.

\section{REFERENCIAS}

1. Organización Mundial de la Salud. Metas mundiales de nutrición 2025: documento normativo sobre bajo peso al nacer [Global nutrition targets 2025: low birth weight policy brief ]. Ginebra:; 2017 (WHO/NMH/NHD/14.5). página consultada el 28 de julio de 2018).

2. Organización Mundial de la Salud. March of Dimes, La Alianza para la Salud de la Madre, el Recién Nacido y el Niño, Save the Children, OMS. Born too soon: the global action report on preterm birth. Ginebra, , 2012 (http:// whqlibdoc.who.int/publications/2012/9789241503433_eng. pdf, página consultada el 28 de julio de 2018).

3. Risnes KR, Vatten LJ, Baker JL, Jameson K, Sovio U, Kajantie E et al. Birthweight and mortality in adulthood: a systematic review and meta-analysis. Int J Epidemiol. 2011; 40:647-61. doi:10.1093/ije/dyq267.

4. Kim D, Saada A. The social determinants of infant mortality and birth outcomes in western developed nations: a cross country systematic review. Int J Environ Res Public Health. 2013; 10(6):2296-335. doi:10.3390/ ijerph10062296

5. Instituto nacional de salud. Ramírez P. Informe final del evento bajo peso al nacer a término, Colombia, 2015.
6. Ramírez P. Informe final del evento de bajo peso al nacer, Instituto nacional de salud, Colombia , 2015 , páginas de 1 al 45

7. Definición de embarazo a término. Rev Obstet Ginecol Venez. 2013;73:285-6.

8. Cabero L. Tratado de ginecología y obstetricia 2a ed. Sociedad española de ginecología y obstetricia SEGO. Barcelona: Panamericana: 2013

9. Decherney A, Nathan L, Luafer N, Roman A. Diagnóstico y tratamiento ginecoobstetricos. $11^{\mathrm{a}}$ edición. México D.F: Lange; 2014.

10. Williams J. Williams Obstetricia, $23^{\mathrm{a}}$ edición ; Ed Mc Graw Hill. Capítulo 36, páginas 804 a 831.

11. Vergara J. Ministerio de Salud y Protección Social, Ruta de Atención Materno Perinatal AMBUQ, 2017. pagínas 1 a 43. 